\title{
ON PERIODIC-TYPE SOLUTIONS OF SYSTEMS OF LINEAR ORDINARY DIFFERENTIAL EQUATIONS
}

\section{KIGURADZE}

Received 1 October 2002

We establish nonimprovable, in a certain sense, sufficient conditions for the existence of a unique periodic-type solution for systems of linear ordinary differential equations.

\section{Formulation of the problem and statement of the main results}

Let $n_{1}$ and $n_{2}$ be natural numbers, $\omega>0, \Lambda_{i} \in \mathbb{R}^{n_{i} \times n_{i}}(i=1,2)$ nonsingular matrices, and $\mathscr{P}_{i k}: \mathbb{R} \rightarrow \mathbb{R}^{n_{i} \times n_{k}}(i, k=1,2)$ and $q_{i}: \mathbb{R} \rightarrow \mathbb{R}^{n_{i}}(i=1,2)$ matrix and vector functions whose components are Lebesgue integrable on each compact interval. We consider the problem on the existence and uniqueness of a solution of the linear differential system

$$
\frac{d x_{i}}{d t}=\mathscr{P}_{i 1}(t) x_{1}+\mathscr{P}_{i 2}(t) x_{2}+q_{i}(t) \quad(i=1,2),
$$

satisfying the conditions

$$
x_{i}(t+\omega)=\Lambda_{i} x_{i}(t) \quad \text { for } t \in \mathbb{R}(i=1,2) .
$$

When $\Lambda_{1}$ and $\Lambda_{2}$ are unit matrices, this problem becomes the well-known problem on a periodic solution which has been the subject of numerous studies (see, e.g., $[1,2,3,4$, $5,6,7,8,9,10,11,12,13,14]$ and the references therein).

In this paper, sufficient conditions for the unique solvability of problem (1.1), (1.2) are established, which are nonimprovable in a certain sense and in particular provide new results on the existence of a unique $\omega$-periodic solution of system (1.1).

The following notation is used in the paper:

(1) $\mathbb{R}$ is the set of real numbers;

(2) $\mathbb{R}^{n}$ is the $n$-dimensional real Euclidean space;

(3) $x=\left(\xi_{i}\right)_{i=1}^{n} \in \mathbb{R}^{n}$ is the column vector with components $\xi_{1}, \ldots, \xi_{n}$,

$$
|x|=\left(\left|\xi_{i}\right|\right)_{i=1}^{n}, \quad\|x\|=\left(\sum_{i=1}^{n} \xi_{i}^{2}\right)^{1 / 2} ;
$$


(4) $x \cdot y$ is the scalar product of vectors $x, y \in \mathbb{R}^{n}$;

(5) $\mathbb{R}^{m \times n}$ is the space of $m \times n$ matrices $X=\left(\xi_{i k}\right)_{i, k=1}^{m, n}$ with components $\xi_{i k}(i=1, \ldots$, $m ; k=1, \ldots, n)$,

$$
|X|=\left(\left|\xi_{i k}\right|\right)_{i, k=1}^{m, n}, \quad\|X\|=\left(\sum_{k=1}^{n} \sum_{i=1}^{m} \xi_{i k}^{2}\right)^{1 / 2} ;
$$

(6) $X^{*}$ is the transposed matrix of the matrix $X$;

(7) $E_{n}$ is the unit $n \times n$ matrix;

(8) $\operatorname{det}(X)$ is the determinant of the matrix $X$;

(9) $r(X)$ is the spectral radius of the matrix $X \in \mathbb{R}^{n \times n}$;

(10) if $X \in \mathbb{R}^{n \times n}$, then $\lambda_{0}(X)$ is a minimal eigenvalue of the matrix $(1 / 2)\left(X+X^{*}\right)$.

Inequalities between the matrices and the vectors are understood componentwise. Throughout the paper, it will be assumed that

$$
\mathscr{P}_{i k}(t+\omega)=\Lambda_{i} \mathscr{P}_{i k}(t) \Lambda_{k}^{-1}, \quad q_{i}(t+\omega)=\Lambda_{i} q_{i}(t) \quad \text { for } t \in \mathbb{R}(i, k=1,2) .
$$

For each $i \in\{1,2\}$, consider the differential system

$$
\frac{d x}{d t}=\mathscr{P}_{i i}(t) x
$$

and denote by $X_{i}$ its fundamental matrix satisfying the initial condition

$$
X_{i}(0)=E_{n_{i}} .
$$

If, however, the matrix $\Lambda_{i}-X_{i}(\omega)$ is nonsingular, then it is assumed that

$$
G_{i}(t, \tau)=X_{i}(t)\left(X_{i}^{-1}(\omega) \Lambda_{i}-E_{n_{i}}\right)^{-1} X_{i}^{-1}(\tau) .
$$

For each $i \in\{1,2\}$, we define a matrix function $\Lambda_{i 0}:[0,3 \omega] \rightarrow \mathbb{R}^{n_{i} \times n_{i}}$ in the following manner:

$$
\begin{gathered}
\Lambda_{i 0}(s)=E_{n_{i}} \quad \text { for } 0 \leq s \leq \omega, \\
\Lambda_{i 0}(s)=\left|\Lambda_{i}^{k}\right| \quad \text { for } k \omega<s \leq(k+1) \omega(k=1,2) .
\end{gathered}
$$

Theorem 1.1. Let

$$
\operatorname{det}\left(\Lambda_{i}-X_{i}(\omega)\right) \neq 0 \quad(i=1,2),
$$

and there exists a nonnegative matrix $A \in \mathbb{R}^{n_{1} \times n_{1}}$ such that $r(A)<1$, and

$$
\int_{t}^{t+\omega} \int_{\tau}^{\tau+\omega}\left|G_{1}(t, \tau) \mathscr{P}_{12}(\tau) G_{2}(\tau, s) \mathscr{P}_{12}(s)\right| \Lambda_{10}(s) d s d \tau \leq A \quad \text { for } 0 \leq t \leq \omega .
$$

Then problem (1.1), (1.2) has a unique solution. 
Example 1.2. Let $n_{1}=n_{2}=1, \Lambda_{1}=\Lambda_{2}=1, q_{i}(t) \equiv 0, \mathscr{P}_{i 1}(t) \equiv p_{i}(t)$, and $\mathscr{P}_{i 2}(t)=-p_{i}(t)$, where $\left.p_{i}: \mathbb{R} \rightarrow\right] 0,+\infty[(i=1,2)$ are the integrable on $[0, \omega] \omega$-periodic functions. Then conditions (1.5), (1.11), and (1.12), where $A=1$, are fulfilled. On the other hand, in the considered case, system (1.1) has the form

$$
\frac{d x_{i}}{d t}=p_{i}(t)\left(x_{1}-x_{2}\right) \quad(i=1,2)
$$

and therefore problem (1.1), (1.2) has an infinite set of solutions

$$
\left\{\left(x_{1}, x_{2}\right): x_{1}(t) \equiv x_{2}(t) \equiv c, c \in \mathbb{R}\right\}
$$

This example shows that the condition $r(A)<1$ in Theorem 1.1 is nonimprovable and it cannot be replaced by the condition $r(A) \leq 1$.

Theorem 1.3. Let

$$
\begin{gathered}
X_{i}(\omega)=\Lambda_{1}, \quad \operatorname{det}\left(\Lambda_{2}-X_{2}(\omega)\right) \neq 0, \\
\operatorname{det}\left(Q_{0}\right) \neq 0,
\end{gathered}
$$

where

$$
\begin{aligned}
Q_{0} & =\int_{0}^{\omega} X_{1}^{-1}(\tau) \mathscr{P}_{12}(\tau) Q(\tau) d \tau, \\
Q(t) & =\int_{t}^{t+\omega} G_{2}(t, s) \mathscr{P}_{21}(s) X_{1}(s) d s .
\end{aligned}
$$

Let, further, there exist a nonnegative matrix $A \in \mathbb{R}^{n_{2} \times n_{2}}$ such that $r(A)<1$, and

$$
\int_{t}^{t+\omega}\left[H(t, \tau)+\int_{\tau}^{\tau+\omega}\left|Q(t) Q_{0}^{-1} X_{1}^{-1}(\tau) \mathscr{P}_{12}(\tau)\right| H(\tau, s) d s\right] d \tau \leq A \quad \text { for } 0 \leq t \leq \omega,
$$

where

$$
H(t, \tau)=\int_{0}^{\tau}\left|G_{2}(t, \tau) \mathscr{P}_{21}(\tau) X_{1}(\tau) X_{1}^{-1}(s) \mathscr{P}_{12}(s)\right| \Lambda_{20}(s) d s .
$$

Then problem (1.1), (1.2) has a unique solution.

Example 1.4. Consider the problem

$$
\begin{array}{ll}
\frac{d x_{1}}{d t}=B_{1} x_{2}, & \frac{d x_{2}}{d t}=\varepsilon B_{2} x_{1}+B x_{2}, \\
x_{i}(t+\omega)=x_{i}(t) & \text { for } t \in \mathbb{R}(i=1,2),
\end{array}
$$

where $\varepsilon$ is a positive constant, $B_{1} \in \mathbb{R}^{n_{1} \times n_{2}}, B_{2} \in \mathbb{R}^{n_{2} \times n_{1}}, B \in \mathbb{R}^{n_{2} \times n_{2}}$, and $\operatorname{det}(B) \neq 0$. This problem is obtained from problem (1.1), (1.2) when $\Lambda_{i}=E_{n_{i}}(i=1,2), \mathscr{P}_{1}$ is a zero matrix, $\mathscr{P}_{12}(t) \equiv B_{1}, \mathscr{P}_{21}(t) \equiv \varepsilon B_{2}, \mathscr{P}_{22}(t) \equiv B$, and $q(t) \equiv 0$. It is obvious that conditions 
(1.5) and (1.15) are fulfilled for this problem. On the other hand, by virtue of (1.17) and (1.19), we have

$$
\begin{gathered}
Q(t) \equiv \varepsilon B^{-1} B_{2}, \quad Q_{0}=\varepsilon \omega B_{1} B^{-1} B_{2}, \\
H(t, \tau)=\tau \varepsilon\left|\left(\exp (-\omega B)-E_{n_{2}}\right)^{-1} \exp ((t-\tau) B) B_{2} B_{1}\right| .
\end{gathered}
$$

Therefore, condition (1.16) is fulfilled if and only if

$$
\operatorname{det}\left(B_{1} B^{-1} B_{2}\right) \neq 0
$$

If the latter inequality is fulfilled, then, by Theorem 1.3 , there exists $\varepsilon_{0}>0$ such that, for arbitrary $\varepsilon \in] 0, \varepsilon_{0}$ [, problem $(1.20)$ has only a trivial solution. If $\operatorname{det}\left(B_{1} B^{-1} B_{2}\right)=0$, then, for arbitrary $\varepsilon$, problem (1.20) has an infinite set of solutions

$$
\left\{\left(x_{1}, x_{2}\right): x_{1}(t) \equiv c x_{10}, x_{2}(t)=c x_{20}, c \in \mathbb{R}\right\}
$$

where $x_{10} \in \mathbb{R}^{n_{1}}$ is the eigenvector of the matrix $B_{1} B^{-1} B_{2}$ corresponding to the zero eigenvalue and $x_{20}=-\varepsilon B^{-1} B_{2} x_{10}$.

Example 1.4 shows that condition (1.16) is essential and cannot be omitted.

Theorem 1.5. Let there exist a matrix $A \in \mathbb{R}^{n_{1} \times n_{2}}$, symmetric matrices $A_{i} \in \mathbb{R}^{n_{i} \times n_{i}}$ $(i=1,2)$, and an integrable function $\delta:[0, \omega] \rightarrow[0,+\infty[$ such that

$$
\Lambda_{2}^{*} A \Lambda_{1}=A, \quad \Lambda_{i}^{*} A_{i} \Lambda_{i}=A_{i} \quad(i=1,2)
$$

and the following inequalities are fulfilled almost everywhere on $[0, \omega]$ :

$$
\begin{gathered}
\lambda_{0}\left(A_{1} \mathscr{P}_{11}(t)+A^{*} \mathscr{P}_{21}(t)\right) \geq \delta(t), \quad \lambda_{0}\left(A_{2} \mathscr{P}_{22}(t)+A \mathscr{P}_{12}(t)\right) \geq \delta(t), \\
\delta(t) \geq p(t),
\end{gathered}
$$

where

$$
p(t)=\frac{1}{2}\left(\left\|A_{1} \mathscr{P}_{12}(t)+A^{*} \mathscr{P}_{22}(t)\right\|+\left\|A_{2} \mathscr{P}_{21}(t)+A \mathscr{P}_{11}(t)\right\|\right) .
$$

If, moreover,

$$
\int_{0}^{\omega}(\delta(t)-p(t)) d t>0
$$

then problem (1.1), (1.2) has a unique solution.

Example 1.2 shows that conditions (1.5), (1.24), (1.25), and (1.26) do not guarantee the unique solvability of problem (1.1), (1.2). Therefore, condition (1.28) in Theorem 1.5 is essential and cannot be omitted. 


\section{Auxiliary propositions}

In this section, we consider the problem

$$
\begin{aligned}
\frac{d x}{d t} & =\mathscr{P}(t) x+q(t), \\
x(t+\omega) & =\Lambda x(t) \quad \text { for } t \in \mathbb{R},
\end{aligned}
$$

assuming that $\Lambda \in \mathbb{R}^{n \times n}$ is a nonsingular matrix, and $\mathscr{P}: \mathbb{R} \rightarrow \mathbb{R}^{n \times n}$ and $q: \mathbb{R} \rightarrow \mathbb{R}^{n}$ are matrix and vector functions with components Lebesgue integrable on $[0, \omega]$ and satisfying the conditions

$$
\begin{aligned}
\mathscr{P}(t+\omega) & =\Lambda \mathscr{P}(t) \Lambda^{-1} \quad \text { for } t \in \mathbb{R}, \\
q(t+\omega) & =\Lambda q(t) \quad \text { for } t \in \mathbb{R} .
\end{aligned}
$$

We denote by $X$ the fundamental matrix of the homogeneous differential system

$$
\frac{d x}{d t}=\mathscr{P}(t) x
$$

satisfying the initial condition

$$
X(0)=E_{n}
$$

Condition (2.3) immediately implies the following lemma.

Lemma 2.1. The matrix function $X$ satisfies the identity

$$
X(t+\omega)=\Lambda X(t) \Lambda^{-1} X(\omega) \quad \text { for } t \in \mathbb{R} .
$$

Lemma 2.2. Problem (2.5), (2.2) has only a trivial solution if and only if

$$
\operatorname{det}(\Lambda-X(\omega)) \neq 0
$$

Proof. Let $x$ be an arbitrary solution of system (2.5). Then

$$
x(t)=X(t) c \quad \text { for } t \in \mathbb{R},
$$

where $c \in \mathbb{R}^{n}$. Hence, by Lemma 2.1, it follows that $x$ is a solution of problem (2.5), (2.2) if and only if

$$
\left(\Lambda X(t)-\Lambda X(t) \Lambda^{-1} X(\omega)\right) c=0 \quad \text { for } t \in \mathbb{R}
$$

However, for the latter identity to be fulfilled, it is necessary and sufficient that $c$ be a solution of the system of algebraic equations

$$
(\Lambda-X(\omega)) c=0
$$

Therefore, problem (2.5), (2.2) has only a trivial solution if and only if the latter system has only a trivial solution, that is, if (2.8) is fulfilled. 
Lemma 2.3. Problem (2.1), (2.2) is uniquely solvable if and only if the corresponding homogeneous problem (2.5), (2.2) has only a trivial solution, that is, if inequality (2.8) is fulfilled. Moreover, if (2.8) is fulfilled, then the solution of problem (2.1), (2.2) admits the representation

$$
x(t)=\int_{t}^{t+\omega} G(t, s) q(s) d s \quad \text { for } t \in \mathbb{R}
$$

where

$$
G(t, s)=X(t)\left(X^{-1}(\omega) \Lambda-E_{n}\right)^{-1} X^{-1}(s)
$$

Proof. By Lemma 2.2, to prove Lemma 2.3, it is sufficient to establish that if inequality (2.8) is fulfilled, then the vector function $x$ given by equality (2.12) is a solution of problem (2.1), (2.2).

According to (2.7) and (2.13), we have

$$
\begin{aligned}
\frac{\partial G(t, s)}{\partial t}= & \mathscr{P}(t) G(t, s) \quad \text { for } s \in \mathbb{R} \text { and almost all } t \in \mathbb{R} \\
G(t, t+\omega) \Lambda-G(t, t)= & X(t)\left(X^{-1}(\omega) \Lambda-E_{n}\right)^{-1}\left(X^{-1}(t+\omega) \Lambda-X^{-1}(t)\right) \\
= & X(t)\left(X^{-1}(\omega) \Lambda-E_{n}\right)^{-1}\left(X^{-1}(\omega) \Lambda-E_{n}\right) X^{-1}(t) \\
= & E_{n} \quad \text { for } t \in \mathbb{R} \\
G(t+\omega, s+\omega)= & \Lambda X(t) \Lambda^{-1} X(\omega)\left(X^{-1}(\omega) \Lambda-E_{n}\right)^{-1} \\
& \times\left(\Lambda^{-1} X(\omega)\right)^{-1} X^{-1}(t) \Lambda^{-1} \\
= & \Lambda G(t, s) \Lambda^{-1} \text { for } s \in \mathbb{R}, t \in \mathbb{R} .
\end{aligned}
$$

If, along with these identities, we also take into consideration condition (2.4), then, from (2.12), we obtain

$$
\begin{aligned}
\frac{d x(t)}{d t} & =\mathscr{P}(t) x(t)+G(t, t+\omega) q(t+\omega)-G(t, t) q(t) \\
& =\mathscr{P}(t) x(t)+(G(t, t+\omega) \Lambda-G(t, t)) q(t) \\
& =\mathscr{P}(t) x(t)+q(t) \quad \text { for almost all } t \in \mathbb{R}^{n}, \\
x(t+\omega) & =\int_{t+\omega}^{t+2 \omega} G(t+\omega, s) q(s) d s=\int_{t}^{t+\omega} G(t+\omega, s+\omega) q(s+\omega) d s \\
& =\Lambda \int_{t}^{t+\omega} G(t, s) q(s) d s=\Lambda x(t) \quad \text { for } t \in \mathbb{R} .
\end{aligned}
$$

Thus $x$ is a solution of problem (2.1), (2.2). 


\section{Proofs of the main results}

Proof of Theorem 1.1. By Lemma 2.3, it is sufficient to show that the homogeneous problem

$$
\begin{gathered}
\frac{d x_{i}}{d t}=\mathscr{P}_{i 1}(t) x_{1}+\mathscr{P}_{i 2}(t) x_{2}, \\
x_{i}(t+\omega)=\Lambda_{i} x_{i}(t) \quad \text { for } t \in \mathbb{R}(i=1,2)
\end{gathered}
$$

has only a trivial solution.

Let $\left(x_{1}, x_{2}\right)$ be an arbitrary solution of this problem. By virtue of Lemma 2.3, condition (1.11) and the equalities

$$
\begin{gathered}
\mathscr{P}_{12}(t+\omega) x_{2}(t+\omega)=\Lambda_{1} \mathscr{P}_{12}(t) x_{2}(t), \\
\mathscr{P}_{21}(t+\omega) x_{1}(t+\omega)=\Lambda_{2} \mathscr{P}_{21}(t) x_{1}(t) \quad \text { for almost all } t \in \mathbb{R}
\end{gathered}
$$

guarantee the validity of the representations

$$
\begin{aligned}
& x_{1}(t)=\int_{t}^{t+\omega} G_{1}(t, s) \mathscr{P}_{12}(s) x_{2}(s) d s, \\
& x_{2}(t)=\int_{t}^{t+\omega} G_{2}(t, s) \mathscr{P}_{21}(s) x_{1}(s) d s .
\end{aligned}
$$

Therefore,

$$
x_{1}(t)=\int_{t}^{t+\omega} \int_{\tau}^{\tau+\omega} G_{1}(t, \tau) \mathscr{P}_{12}(\tau) G_{2}(\tau, s) \mathscr{P}_{21}(s) x_{1}(s) d s
$$

Let

$$
\begin{gathered}
x_{1}(t)=\left(x_{1 k}(t)\right)_{k=1}^{n_{1}}, \\
\rho_{k}=\max \left\{\left|x_{1 k}(t)\right|: 0 \leq t \leq \omega\right\} \quad\left(k=1, \ldots, n_{1}\right), \rho=\left(\rho_{k}\right)_{k=1}^{n_{1}} .
\end{gathered}
$$

Then by (1.9), (1.10) for $i=1$, we have

$$
\left|x_{1}(s)\right| \leq \Lambda_{10}(s) \rho \quad \text { for } 0 \leq s \leq 3 \omega .
$$

If, along with this, we also take into consideration inequality (1.12), then, from representation (3.5), we obtain

$$
\left|x_{1}(t)\right| \leq A \rho \text { for } 0 \leq t \leq \omega \text {. }
$$

Hence $\rho \leq A \rho$ and, therefore,

$$
\left(E_{n_{1}}-A\right) \rho \leq 0
$$

According to the condition $r(A)<1$ and the nonnegativeness of the matrix $A$, the matrix $E_{n_{1}}-A$ is nonsingular and $\left(E_{n_{1}}-A\right)^{-1}$ is nonnegative. Hence the multiplication of the 
latter vector inequality by $\left(E_{n_{1}}-A\right)^{-1}$ gives $\rho \leq 0$. Therefore, $\rho=0$, that is,

$$
x_{1}(t)=0 \quad \text { for } 0 \leq t \leq \omega .
$$

By virtue of this equality, from (3.4), it follows that $x_{i}(t)=0$ for $t \in \mathbb{R}(i=1,2)$.

Proof of Theorem 1.3. Let $\left(x_{1}, x_{2}\right)$ be an arbitrary solution of problem (3.1), (3.2). Then by the Cauchy formula, we have

$$
x_{1}(t)=X_{1}(t) c+\int_{0}^{t} X_{1}(t) X_{1}^{-1}(\tau) \mathscr{P}_{12}(\tau) x_{2}(\tau) d \tau,
$$

where $c \in \mathbb{R}^{n_{1}}$. On the other hand, by Lemma 2.3, the nonsingularity of the matrix $\Lambda_{2}-$ $X_{2}(\omega)$ and the equality

$$
\mathscr{P}_{21}(t+\omega) x_{1}(t+\omega)=\Lambda_{2} \mathscr{P}_{21}(t) x_{1}(t) \quad \text { for almost all } t \in \mathbb{R}
$$

guarantee the validity of the representation

$$
x_{2}(t)=\int_{t}^{t+\omega} G_{2}(t, \tau) \mathscr{P}_{21}(\tau) x_{1}(\tau) d \tau
$$

Hence, by virtue of equalities (1.17) and (3.11), it follows that

$$
x_{2}(t)=Q(t) c+\int_{t}^{t+\omega} z(t, \tau) d \tau
$$

where

$$
z(t, \tau)=\int_{0}^{\tau} G_{2}(t, \tau) \mathscr{P}_{21}(\tau) X_{1}(\tau) X_{1}^{-1}(s) \mathscr{P}_{12}(s) x_{2}(s) d s .
$$

By Lemma 2.1 and the equality $X_{1}(\omega)=\Lambda_{1}$, we have

$$
X_{1}(t+\omega)=\Lambda_{1} X(t) \quad \text { for } t \in \mathbb{R} .
$$

Therefore, from (3.11), we find

$$
x_{1}(t+\omega)=\Lambda_{1} X_{1}(t) c+\Lambda_{1} \int_{0}^{t+\omega} X_{1}(t) X_{1}^{-1}(\tau) \mathscr{P}_{12}(\tau) x_{2}(\tau) d \tau .
$$

Hence, by (3.2), it follows that

$$
x_{1}(t)=X_{1}(t) c+\int_{0}^{t+\omega} X_{1}(t) X_{1}^{-1}(\tau) \mathscr{P}_{12}(\tau) x_{2}(\tau) d \tau .
$$

If now we again apply representation (3.11), then it becomes clear that the identity

$$
\int_{t}^{t+\omega} X_{1}^{-1}(\tau) \mathscr{P}_{12}(\tau) x_{2}(\tau) d \tau=0 \quad \text { for } t \in \mathbb{R}
$$

is valid. 
Using (3.14), from the latter identity, we find

$$
\widetilde{Q}(t) c=-\int_{t}^{t+\omega} \int_{\tau}^{\tau+\omega} X_{1}^{-1}(\tau) \mathscr{P}_{12}(\tau) z(\tau, s) d s d \tau,
$$

where

$$
\widetilde{Q}(t)=\int_{t}^{t+\omega} X_{1}^{-1}(\tau) \mathscr{P}_{12}(\tau) Q(\tau) d \tau
$$

By Lemma 2.1,

$$
G_{2}(t+\omega, s+\omega)=\Lambda_{2} G_{2}(t, s) \Lambda_{2}^{-1}
$$

If, along with this identity, we also take into account identities (1.5) and (3.16), then we obtain

$$
Q(t+\omega)=\int_{t}^{t+\omega} G_{2}(t+\omega, s+\omega) \mathscr{P}_{21}(s+\omega) X_{1}(s+\omega) d s=\Lambda_{2} Q(t) .
$$

Therefore, from (1.17) and (3.21), we have

$$
\begin{aligned}
\widetilde{Q}(t)= & \int_{t}^{\omega} X_{1}^{-1}(\tau) \mathscr{P}_{12}(\tau) Q(\tau) d \tau \\
& +\int_{0}^{t} X_{1}^{-1}(\tau+\omega) \mathscr{P}_{12}(\tau+\omega) Q(\tau+\omega) d \tau \\
= & \int_{t}^{\omega} X_{1}^{-1}(\tau) \mathscr{P}_{12}(\tau) Q(\tau) d \tau \\
& +\int_{0}^{t} X_{1}^{-1}(\tau) \mathscr{P}_{12}(\tau) Q(\tau) d \tau=Q_{0} \quad \text { for } t \in \mathbb{R} .
\end{aligned}
$$

By virtue of this fact and condition (1.16), from (3.11), (3.14), and (3.20), we get

$$
\begin{aligned}
x_{1}(t)= & \int_{0}^{t} X_{1}(t) X_{1}^{-1}(\tau) \mathscr{P}_{12}(\tau) x_{2}(\tau) d \tau \\
& -X_{1}(t) \int_{0}^{\omega} \int_{\tau}^{\tau+\omega} Q_{0}^{-1} X_{1}^{-1}(\tau) \mathscr{P}_{12}(\tau) z(\tau, s) d s d \tau, \\
x_{2}(t)= & \int_{t}^{t+\omega}\left(z(t, \tau)-\int_{\tau}^{\tau+\omega} Q(t) Q_{0}^{-1} X_{1}^{-1}(\tau) \mathscr{P}_{12}(\tau) z(\tau, s) d s\right) d \tau .
\end{aligned}
$$

Let $x_{2}(t)=\left(x_{2 k}(t)\right)_{k=1}^{n_{2}}$,

$$
\rho_{k}=\max \left\{\left|x_{2 k}(t)\right|: 0 \leq t \leq \omega\right\} \quad\left(k=1, \ldots, n_{2}\right), \rho=\left(\rho_{k}\right)_{k=1}^{n_{2}} .
$$

Then, by (1.9), (1.10) for $i=2$, we have

$$
\left|x_{2}(s)\right| \leq \Lambda_{20}(s) \rho \quad \text { for } 0 \leq s \leq 3 \omega .
$$

By this inequality and the notation (1.19) and (3.15), we have

$$
|z(t, \tau)| \leq H(t, \tau) \rho \quad \text { for } t \in \mathbb{R}, 0 \leq \tau \leq 3 \omega .
$$


Due to this estimate and inequality (1.18), from (3.26), we find

$$
\left|x_{2}(t)\right| \leq A \rho \quad \text { for } 0 \leq t \leq \omega \text {. }
$$

Hence it is clear that $\rho \leq A \rho$ and, therefore,

$$
\left(E_{n_{2}}-A\right) \rho \leq 0
$$

By virtue of the condition $r(A)<1$ and the nonnegativeness of the matrix $A$, the latter inequality implies $\rho=0$. Therefore,

$$
x_{2}(t)=0, \quad z(t, \tau)=0 \quad \text { for } 0 \leq t \leq \omega, 0 \leq \tau \leq 3 \omega,
$$

due to which we find from (3.2) and (3.25) that $x_{i}(t)=0$ for $t \in \mathbb{R}(i=1,2)$. Thus problem (3.1), (3.2) has only a trivial solution. By Lemma 2.3, this fact guarantee the unique solvability of problem (1.1), (1.2).

Proof of Theorem 1.5. By virtue of Lemma 2.3, it is sufficient to establish that problem (3.1), (3.2) has only a trivial solution.

Let $\left(x_{1}, x_{2}\right)$ be an arbitrary solution of problem (3.1), (3.2) and

$$
u(t)=\frac{1}{2}\left(A_{1} x_{1}(t) \cdot x_{1}(t)+A_{2} x_{2}(t) \cdot x_{2}(t)\right)+A x_{1}(t) \cdot x_{2}(t)
$$

Then

$$
\begin{aligned}
u^{\prime}(t)= & A_{1} x_{1}^{\prime}(t) \cdot x_{1}(t)+A_{2} x_{2}^{\prime}(t) \cdot x_{2}(t)+A x_{1}^{\prime}(t) \cdot x_{2}(t)+A^{*} x_{2}^{\prime}(t) \cdot x_{1}(t) \\
= & \left(A_{1} \mathscr{P}_{11}(t)+A^{*} \mathscr{P}_{21}(t)\right) x_{1}(t) \cdot x_{1}(t) \\
& +\left(A_{2} \mathscr{P}_{22}(t)+A \mathscr{P}_{12}(t)\right) x_{2}(t) \cdot x_{2}(t)+\left(A_{1} \mathscr{P}_{12}(t)+A^{*} \mathscr{P}_{22}(t)\right) x_{2}(t) \cdot x_{1}(t) \\
& +\left(A_{2} \mathscr{P}_{21}(t)+A \mathscr{P}_{11}(t)\right) x_{1}(t) \cdot x_{2}(t) \quad \text { for almost all } t \in \mathbb{R} .
\end{aligned}
$$

However, by conditions (1.25) and the Schwartz inequality, for almost all $t \in[0, \omega]$, we have

$$
\begin{gathered}
\left(A_{1} \mathscr{P}_{11}(t)+A^{*} \mathscr{P}_{21}(t)\right) x_{1}(t) \cdot x_{1}(t) \geq \delta(t)\left\|x_{1}(t)\right\|^{2}, \\
\left(A_{2} \mathscr{P}_{22}(t)+A \mathscr{P}_{12}(t)\right) x_{2}(t) \cdot x_{2}(t) \geq \delta(t)\left\|x_{2}(t)\right\|^{2}, \\
\left(A_{1} \mathscr{P}_{12}(t)+A^{*} \mathscr{P}_{22}(t)\right) x_{2}(t) \cdot x_{1}(t)+\left(A_{2} \mathscr{P}_{21}(t)+A \mathscr{P}_{11}(t)\right) x_{1}(t) \cdot x_{2}(t) \\
\leq 2 p(t)\left\|x_{1}(t)\right\|\left\|x_{2}(t)\right\| \leq p(t)\left(\left\|x_{1}(t)\right\|^{2}+\left\|x_{2}(t)\right\|^{2}\right),
\end{gathered}
$$

where $p$ is the function given by equality (1.27). Therefore,

$$
u^{\prime}(t) \geq(\delta(t)-p(t))\left(\left\|x_{1}(t)\right\|^{2}+\left\|x_{2}(t)\right\|^{2}\right) \quad \text { for almost all } t \in[0, \omega]
$$


On the other hand, by virtue of (1.24) and (3.2), we have

$$
\begin{aligned}
u(\omega)= & \frac{1}{2}\left(A_{1} \Lambda_{1} x_{1}(0) \cdot \Lambda_{1} x_{1}(0)+A_{2} \Lambda_{2} x_{2}(0) \cdot \Lambda_{2} x_{2}(0)\right) \\
& +A \Lambda_{1} x_{1}(0) \cdot \Lambda_{2} x_{2}(0) \\
= & \frac{1}{2}\left(\Lambda_{1}^{*} A_{1} \Lambda_{1} x_{1}(0) \cdot x_{1}(0)+\Lambda_{2}^{*} A_{2} \Lambda_{2} x_{2}(0) \cdot x_{2}(0)\right) \\
& +\Lambda_{2}^{*} A \Lambda_{1} x_{1}(0) \cdot x_{2}(0)=u(0) .
\end{aligned}
$$

Thus

$$
0=\int_{0}^{\omega} u^{\prime}(t) d t \geq \int_{0}^{\omega}(\delta(t)-p(t))\left(\left\|x_{1}(t)\right\|^{2}+\left\|x_{2}(t)\right\|^{2}\right) d t .
$$

Hence, by virtue of conditions (1.26) and (1.28), it follows that there exists $t_{0} \in[0, \omega]$ such that

$$
x_{i}\left(t_{0}\right)=0 \quad(i=1,2)
$$

Therefore, $x_{i}(t)=0$ for $t \in \mathbb{R}(i=1,2)$ since system (3.1) with the zero initial conditions has only a trivial solution.

\section{Acknowledgment}

This work was supported by the INTAS Grant 00136.

\section{References}

[1] R. E. Gaines and J. L. Mawhin, Coincidence Degree, and Nonlinear Differential Equations, Lecture Notes in Mathematics, vol. 568, Springer-Verlag, New York, 1977.

[2] I. Kiguradze, Periodic solutions of systems of nonautonomous ordinary differential equations, Mat. Zametki 39 (1986), no. 4, 562-575 (Russian), translated in Math. Notes 39 (1986), 308315.

[3] Boundary value problems for systems of ordinary differential equations, Current Problems in Mathematics. Newest Results, Itogi Nauki i Tekhniki, vol. 30, Akad. Nauk SSSR Vsesoyuz. Inst. Nauchn. i Tekhn. Inform., Moscow, 1987, pp. 3-103, translated in J. Soviet Math. 43 (1988), no.2, 2259-2339 (Russian).

[4] The Initial Value Problem and Boundary Value Problems for Systems of Ordinary Differential Equations. Vol. I., Metsniereba, Tbilisi, 1997 (Russian).

[5] M. A. Krasnosel'skiĭ, On the theory of periodic solutions to non-autonomous differential equations, Uspehi Mat. Nauk 21 (1966), no. 3 (129), 53-74 (Russian), translated in Russian Math. Surveys 21 (1966), 53-74.

[6] The Operator of Translation along the Trajectories of Differential Equations, Nauka, Moscow, 1966 (Russian).

[7] M. A. Krasnosel'skii and A. I. Perov, On a certain principle of existence of bounded, periodic and almost periodic solutions of systems of ordinary differential equations, Dokl. Akad. Nauk SSSR 123 (1958), 235-238 (Russian).

[8] A. Lasota and Z. Opial, Sur les solutions périodiques des équations différentielles ordinaires, Ann. Polon. Math. 16 (1964), no. 1, 69-94 (French).

[9] J. L. Massera, The existence of periodic solutions of systems of differential equations, Duke Math. J. 17 (1950), 457-475. 
[10] J. Mawhin, Continuation theorems and periodic solutions of ordinary differential equations, Topological Methods in Differential Equations and Inclusions (Montreal, PQ, 1994), NATO Adv. Sci. Inst. Ser. C Math. Phys. Sci., vol. 472, Kluwer Academic Publishers, Dordrecht, 1995, pp. 291-375.

[11] V. A. Pliss, Nonlocal Problems of the Oscillation Theory, Nauka, Moscow, 1964 (Russian).

[12] K. Schmitt, Periodic solutions of nonlinear differential systems, J. Math. Anal. Appl. 40 (1972), no. $1,174-182$.

[13] T. Yoshizawa, Stability Theory and the Existence of Periodic Solutions and Almost Periodic Solutions, Applied Mathematical Sciences, vol. 14, Springer-Verlag, New York, 1975.

[14] F. Zanolin, Continuation theorems for the periodic problem via the translation operator, Rend. Sem. Mat. Univ. Politec. Torino 54 (1996), no. 1, 1-23.

I. Kiguradze: A. Razmadze Mathematical Institute, Georgian Academy of Sciences, Tbilisi 0193, Georgia

E-mail address: kig@rmi.acnet.ge 


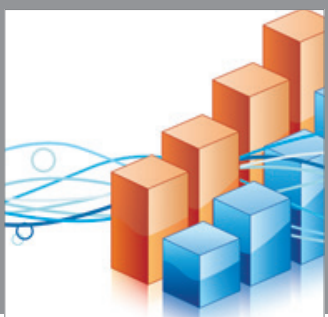

Advances in

Operations Research

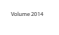

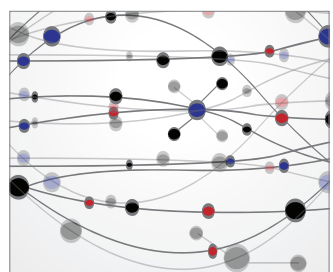

\section{The Scientific} World Journal
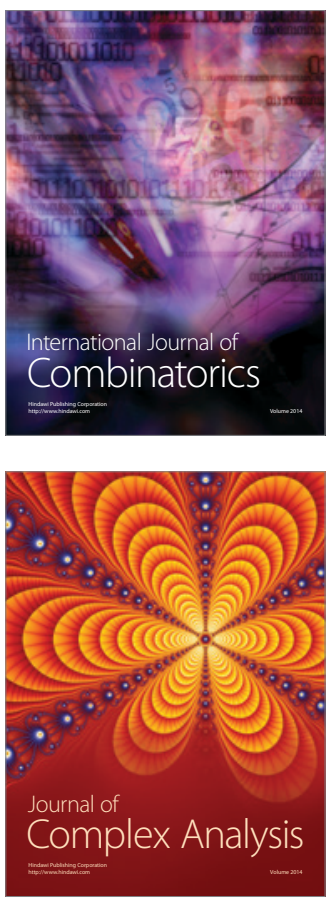

International Journal of

Mathematics and

Mathematical

Sciences
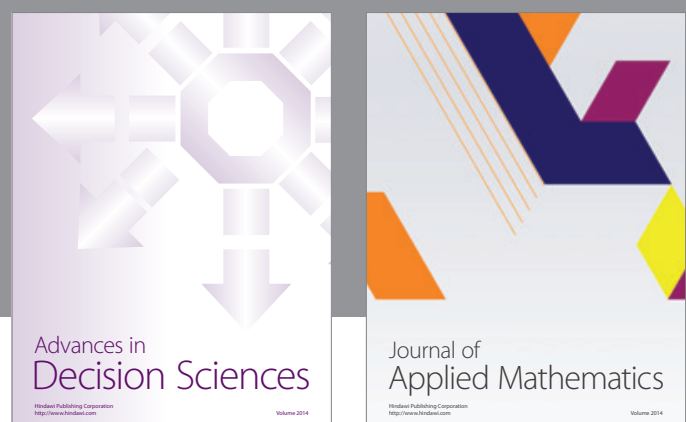

Journal of

Applied Mathematics
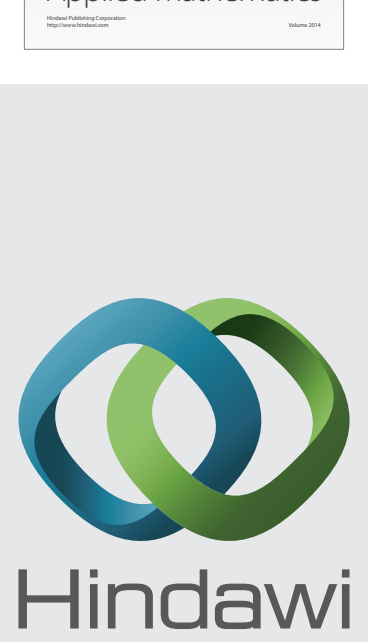

Submit your manuscripts at http://www.hindawi.com
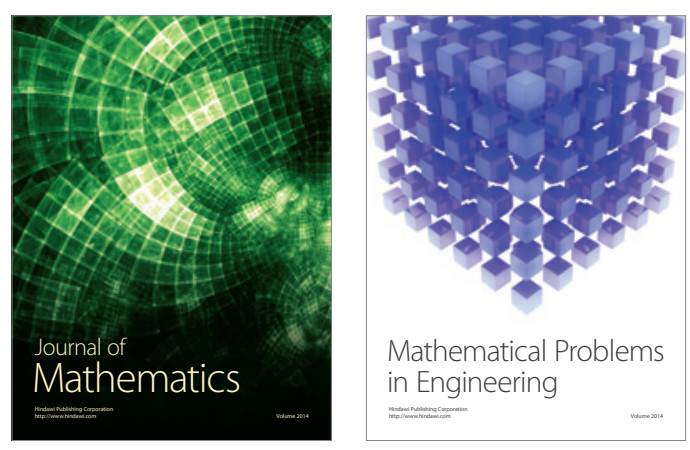

Mathematical Problems in Engineering
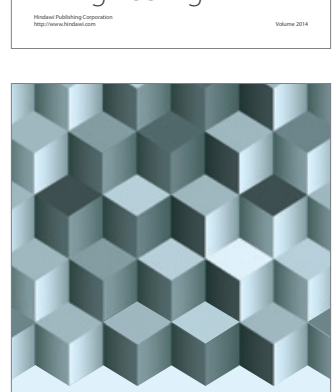

Journal of

Function Spaces
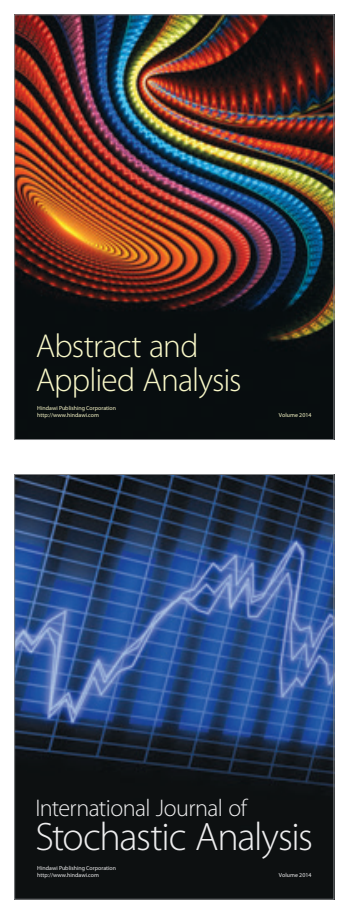

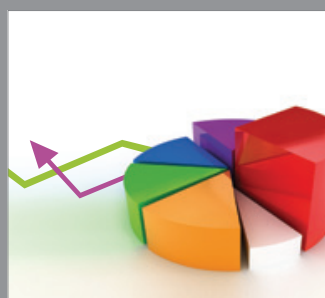

ournal of

Probability and Statistics

Promensencen
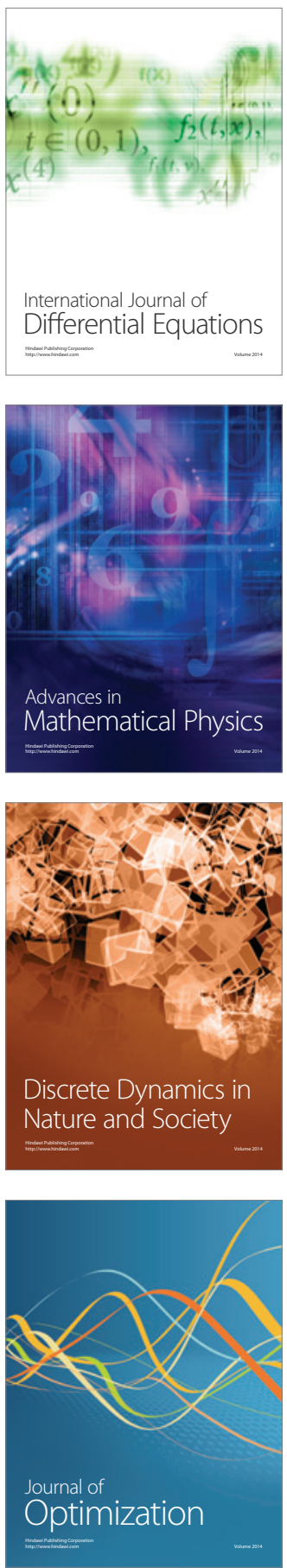